\title{
A Numerical Study of the Chemo-Dynamical Evolution of Elliptical Galaxies
}

\author{
Daisuke Kawata and Brad K. Gibson \\ Centre for Astrophysics and Supercomputing, Swinburne University of \\ Technology, Hawthorn VIC 3122, Australia
}

\begin{abstract}
We investigate the chemo-dynamical evolution of elliptical galaxies, to understand the origin of the mass-dependence of photometric properties such as the colour-magnitude relation (CMR). Our threedimensional TREE N-body/SPH numerical simulation takes into account both Type II and Type Ia supernovae and follows the evolution of the abundances of several chemical elements. We derive the photometric properties of the simulation end-products and compare them with the observed CMR.
\end{abstract}

\section{Introduction}

The colour-magnitude relation (CMR) of elliptical galaxies is a natural byproduct of galaxy formation scenarios involving galactic wind. However, those scenarios were initially based upon a simple model which ignored the internal structure and complex star formation history inherent to galaxy formation. Numerical simulations are a powerful complement which allows one to treat complex physical processes in galaxy formation self-consistently. We have developed a code that can calculate the dynamical, chemical, and photometric evolution of galaxies self-consistently. To understand further the physics of elliptical galaxy formation, we examine whether realistic numerical simulations can reproduce the observed CMR.

\section{Methods and Results}

Our simulations model galaxy formation as an evolution of a low-spin top-hat over-dense sphere, namely a seed galaxy (Kawata 2001). Since we are interested in the evolution of a seed galaxy with different masses, we simulate representative $L^{*}$ and sub- $L^{*}$ seed galaxies with masses of $4 \times 10^{12} M_{\odot}$ and $2 \times 10^{11} M_{\odot}$, respectively. The dynamics of collisionless dark matter and stars is calculated using a Tree N-body code, and the gas component is modeled using SPH. The code includes radiative cooling, star formation, supernova feedback, and metal enrichment. We take into account both Type II (SNe II) and Type Ia (SNe Ia) supernovae ( $\mathrm{SNe}$ ), and chemical enrichment from intermediate mass stars. We assume that $\mathrm{SNe}$ feedback is released as thermal $\left(E_{\mathrm{th}}\right)$ and kinetic $\left(E_{\mathrm{kin}}\right)$ energy, and their ratio is given by a parameter $f_{v}=E_{\text {kin }} /\left(E_{\text {th }}+E_{\text {kin }}\right)$. This parameter controls the magnitude of the effect of SNe. To explore this effect, we carry out 

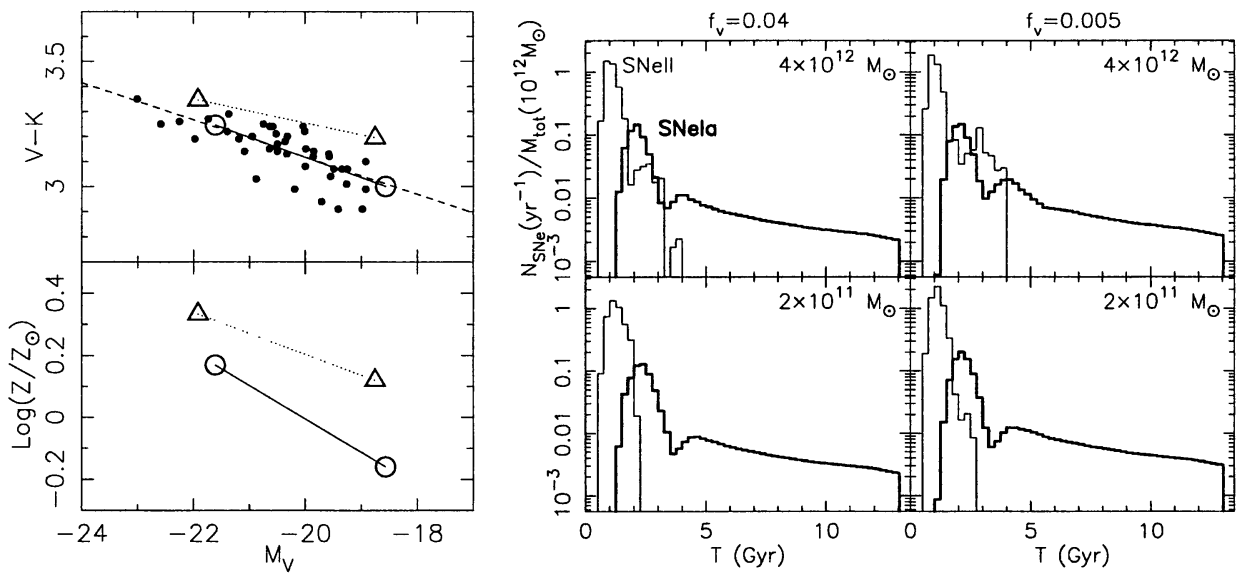

Figure 1. Upper left panel: comparison of the CMRs for the simulation end-products and the Coma cluster galaxies (small dots). Lower left panel: the metallicities against the absolute $V$ band magnitude. The triangles (circles) connected by dotted (solid) lines indicate the results of models with $f_{v}=0.04\left(f_{v}=0.005\right)$. Right panels: time variations of the event rate of SNe II (thin lines) and SNe Ia (thick lines) for all the models.

simulations with strong $\left(f_{v}=0.04\right)$ and weak feedback $\left(f_{v}=0.005\right)$. Figure 1 shows the comparison of the $V-K$ CMR for simulation end-products and Coma cluster galaxies (Bower et al. 1992). These results are obtained by coupling to the population synthesis package of KA97 (Kodama \& Arimoto 1997). The slope of the strong feedback model is steeper and in better agreement with the observed slope than that of the weak feedback model. Figure 1 also shows the metallicities for the models and demonstrates that the CMR is driven by metallicity effects. The history of SNe II, which roughly traces the star formation history, shows that star formation stops abruptly at an early epoch (right panels in Fig. 1). This cessation of star formation is caused by the galactic wind. The galactic wind suppresses further chemical enrichment and leads to bluer colours especially in the low mass system. Moreover, as seen in the CMR, the strong feedback enhances the mass dependence of the suppression of chemical enrichment and causes the steeper slope in the CMR. We find that the strong effect of SNe feedback is required to explain the observed CMR. We also found that SNe Ia play a crucial role in driving and maintaining a galactic wind, and therefore in contributing to the evolution of elliptical galaxies.

\section{References}

Bower, R.G., Lucey, J.R., \& Ellis R.S. 1992, MNRAS, 254, 589

Kawata, D. 2001, ApJ, 558, 598

Kodama, T., \& Arimoto, N. 1997, A\&A, 320, 41 